\title{
Divergent Total Syntheses of Flavonoid Natural Products Isolated From Rosa rugosa and Citrus unshiu
}

\author{
Tze Jing Sum \\ Tze Han Sum \\ Warren R. J. D. Galloway \\ David R. Spring*
}

Department of Chemistry, University of Cambridge, Lensfield Road, Cambridge, CB2 1EW, UK

spring@ch.cam.ac.uk<smiles>[R]c1ccc2c(c1)C(=O)/C(=C/c1ccccc1)O2</smiles><smiles>COc1ccc(OC)c(-c2oc3c(OC)c(OC)c(OC)cc3c(=O)c2OC)c1</smiles>

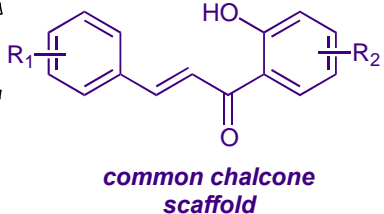<smiles>[R]c1ccc(C(C)=O)c(O)c1</smiles>

readily available starting materials

\section{Received:
Accepted:
Published onlin :}

Abstract The concise and step-economical total syntheses of three hydroxyaurones and one polymethoxyflavone from readily available starting materials is described. A divergent synthetic strategy was employed, which centered on a common chalcone scaffold from which both the aurone and flavone frameworks could be accessed through the use of different oxidative cyclization methods. These are the first reported total syntheses of these biologically interesting compounds.

Key words Flavonoids, Hydroxyaurones, Chalcones, Divergent, Tota Synthesis, Natural Products

Aurones $^{1}$ and flavones ${ }^{2}$ are two sub-classes of the flavonoid family of natural products. ${ }^{3}$ Flavonoids have been found to exhibit a diverse array of interesting biological properties such as anti-cancer, ${ }^{4}$ anti-malaria, ${ }^{5}$ anti-inflammatory, ${ }^{6}$ anti-oxidant, ${ }^{7}$ anti-bacterial $^{8}$ and anti-fungal ${ }^{9}$ activities. Unsurprisingly therefore, natural flavonoids have attracted considerable interest from both the synthetic and medicinal chemistry communities. ${ }^{10}$

In 2012, Gao et al. reported the isolation and characterization of three new aurones, named rugaurones A-C (1-3) from the flowers of Rosa rugosa (Figure 1). ${ }^{11}$ These compounds were found to have promising cytotoxic and anti-HIV properties. In the same year, Shin et al. reported the isolation of three previously unreported flavonoids from the peels of mature fruits Citrus unshiu Marcow (Rutaceae), one of which was named 3,6,7,8,2',5'-hexamethoxyflavone (4). ${ }^{12}$ These peels have been used in traditional Chinese medicine and some isolates from $C$. unshiu have been reported to exhibit anti-proliferative effects. Given the interesting biological profiles of compounds 14 (and the diverse array of properties typically associated with flavonoids in general) we were interested in a more extensive assessment of their biological activities. In view of the very low isolation yields of 1-4 from natural sources, it was thought that

sufficient amounts of materials for biological screening studies could only be secured by total synthesis. Herein, we report the first total syntheses of compounds $\mathbf{1 - 4}$, which were achieved in a step-efficient fashion by the application of divergent and concise synthetic strategy.<smiles>[R6]c1ccc(/C=C2\Oc3cc(OC)c(Br)cc3C2=O)cc1</smiles>

Rugaurone $A$ (1); $\mathrm{R}_{1}=\mathrm{OMe}, \mathrm{R}_{2}=\mathrm{OH}$ Rugaurone $B(2) ; \mathrm{R}_{1}=\mathrm{OH}, \mathrm{R}_{2}=\mathrm{OH}$ Rugaurone C (3); $\mathrm{R}_{1}=\mathrm{OH}, \mathrm{R}_{2}=\mathrm{OMe}$

Figure 1 Naturally occurring hydroxyaurones 1-3 isolated from Rosa rugosa and a 5-deoxyflavone (4) isolated from Citrus unshiu.

Based on our previous work on flavonoid synthesis, ${ }^{13}$ we envisaged a divergent strategy for the construction of natural products 1-4 which used a chalcone scaffold (of the general form 5) as the key branch point (Scheme 1). It was anticipated that both the aurone and flavone frameworks (general structures $\mathbf{6}$ and $\mathbf{7}$ respectively) could be accessed from $\mathbf{5}$ by the application of different oxidation methods; ${ }^{4}$ mercury(II) acetate mediated oxidative cyclization of chalcones $\mathbf{5}$ would yield aurones 6, ${ }^{1}$ whereas Algar-Flynn-Oyamada oxidation with $\mathrm{H}_{2} \mathrm{O}_{2}$ under alkaline conditions would furnish the 5-deoxyflavone framework 7.4 Based on our previous study, ${ }^{13}$ it was presumed that the chalcone precursors $\mathbf{5}$ could be easily accessed via a Claisen-Schmidt aldol condensation between benzaldehydes 8 and acetophenones $\mathbf{9 . 4}$ 


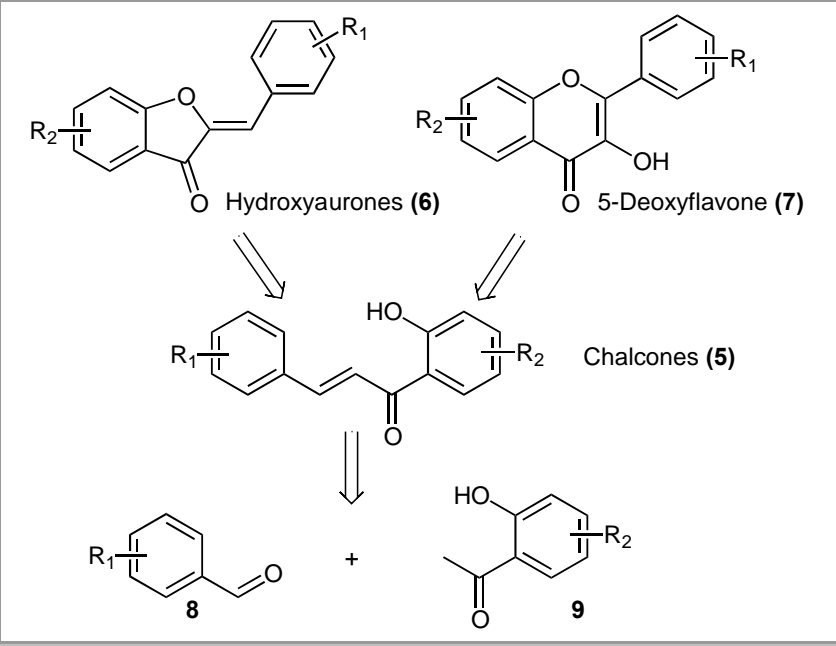

Scheme 1 Retrosynthetic analysis of the hydroxyaurones 6 and 5deoxyflavone 7 .

The total syntheses of rugaurones A-C (1-3) was achieved by the application of this divergent synthetic strategy (Schemes 2 and 3). Benzaldehyde precursor $\mathbf{1 1}$ was prepared from commercially available 4-hydroxybenzaldehyde (10) by MOM protection (Scheme 2). In parallel, the acetophenone building block 13 was accessed from 3,4-dimethoxyphenol (12). ClaisenSchmidt aldol condensation of $\mathbf{1 1}$ and $\mathbf{1 3}$ proceeded smoothly to afford the key chalcone intermediate 14.4,14 Intramolecular mercury(II) acetate mediated oxidative cyclization ${ }^{1}$ produced the intermediate aurone $\mathbf{1 5}$ in high yield and subsequent acidmediated MOM deprotection afforded the desired natural product rugaurone $\mathrm{A}(\mathbf{1})$.

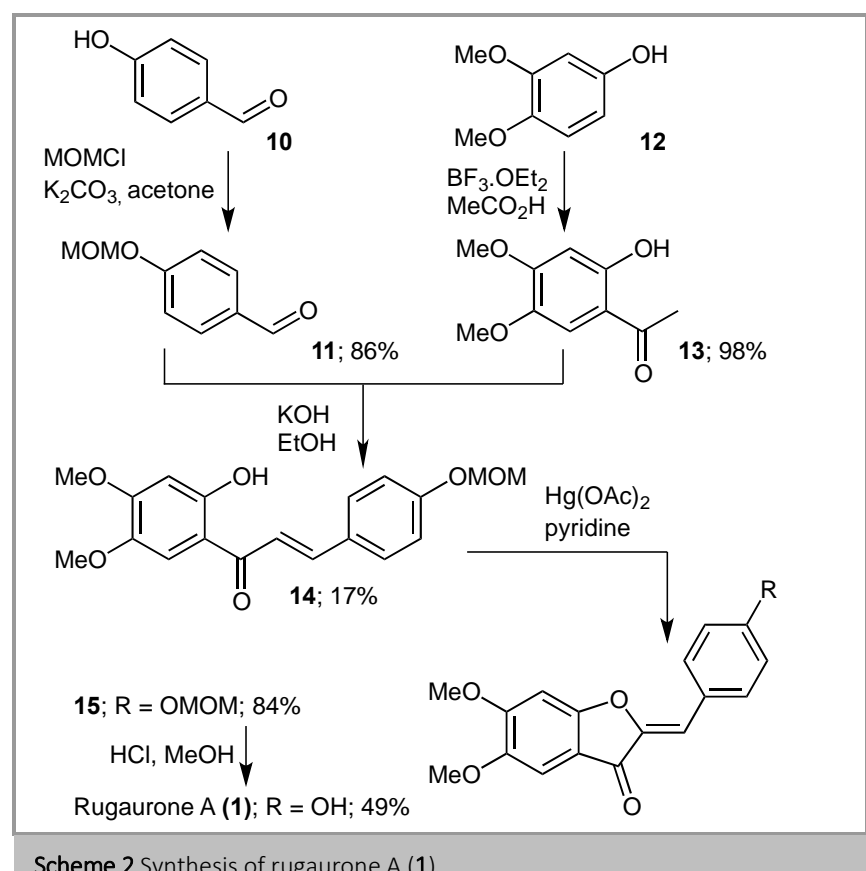

Scheme 2 Synthesis of rugaurone $A(1)$

Rugaurones B (2) and C (3) were prepared in a similarly efficient manner (Scheme 3). Starting from the commercially available hydroquinone 16, acetophenone 17 was synthesized in high yield. Regioselective MOM protection of $\mathbf{1 7}$ afforded 18, which was condensed with the corresponding aldehydes 19-20 under basic ethanolic conditions to give the required MOM protected chalcones 21-22 respectively. Subsequent oxidative cyclization with mercury(II) acetate in pyridine furnished the corresponding aurones 23-24 and MOM deprotection yielded the desired natural products rugaurones B (2) and C (3)

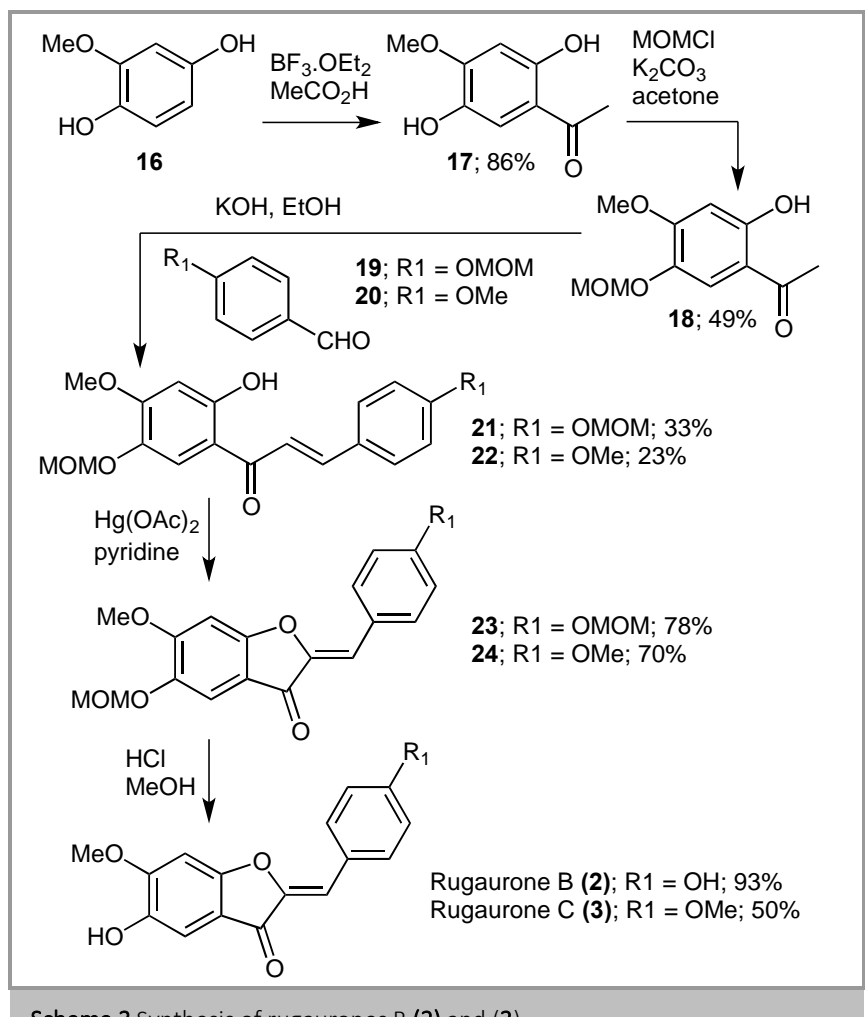

Scheme 3 Synthesis of rugaurones B (2) and (3).

Attention was next turned towards the preparation of the polymethoxyflavone 4 (Scheme 4).

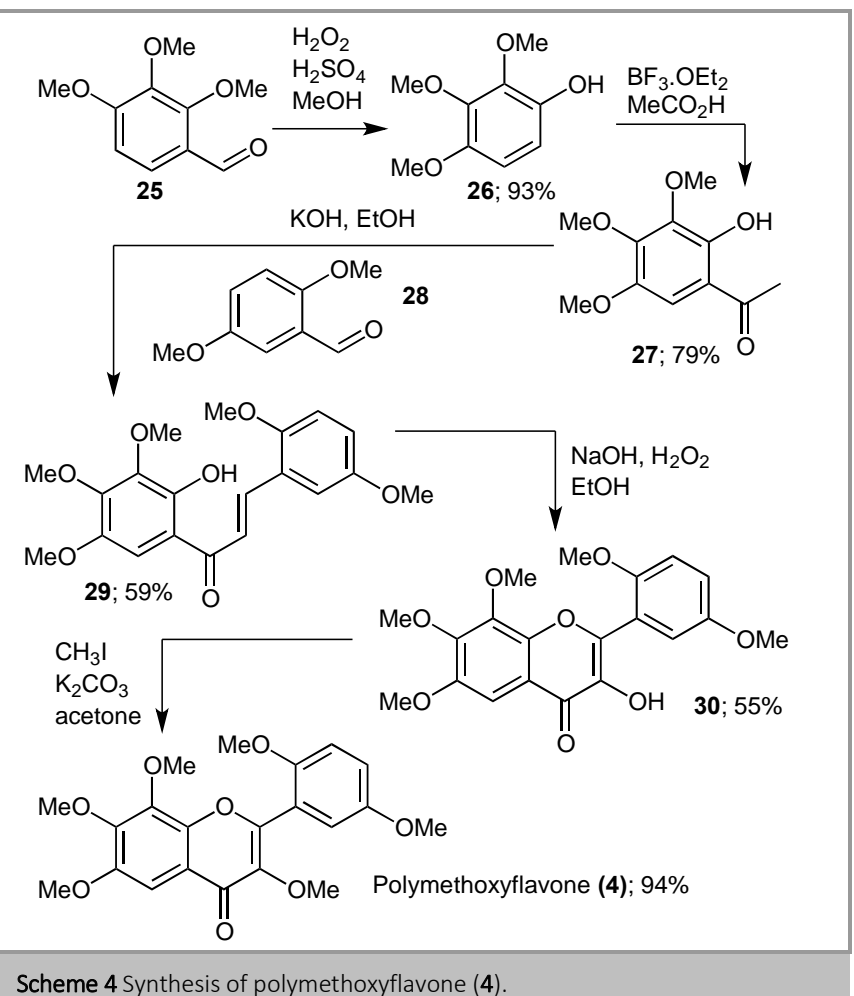


The synthesis commenced with the oxidation of benzaldehyde 25 to phenol 26. Subsequent acylation furnished acetophenone 27 in a good yield. ${ }^{15}$ A Claisen-Schmidt aldol condensation between $\mathbf{2 7}$ and aldehyde $\mathbf{2 8}$ furnished chalcone $\mathbf{2 9}^{2}$ and AlgarFlynn-Oyamada oxidation ${ }^{4}$ yielded the corresponding flavonol scaffold 30. Methylation of $\mathbf{3 0}$ with methyl iodide in acetone under reflux then afforded the desire polymethoxyflavone 4 in an excellent yield.

The divergent strategy employed in the synthesis of natural products 1-4 centres on the selective conversion of chalcone intermediates into aurones or flavones through the use of different oxidation procedures. Attempts to rationalise this selectivity are complicated by uncertainties regarding the mechanisms of these oxidations. It has been suggested that Algar-Flynn oxidation to form the flavone scaffold may proceed by direct intramolecular attack of the phenol oxygen on the alkene (presumably a conjugate addition process) or via an intermediate epoxide derivative; ${ }^{16-18}$ it is possible that stereoelectronic factors play a role in dictating the regioselectivity in both cases, though these are difficult to delineate ${ }^{18,19}$. The exact mechanistic details of the mercury (II) acetate-mediated oxidative cyclisation of chalcones to aurones are also unknown. Most evidence points towards a mechanism which involves formation and cyclisation of an aryloxy-mercury (II) acetate species, ${ }^{20-23}$ rather than an electrophilic-addition pathway (involving activation of the alkene bond by the mercury species and hydroxyl participation $)^{20}$. It has been suggested that 5-membered ring formation from the aryloxymercury (II) acetate species (which leads to the aurone scaffold) is favoured over 6-ring formation (which leads to the flavone scaffold) due to steric effects. ${ }^{23}$

In conclusion, the first total syntheses of the natural products rugaurones A-C (1-3) and polymethoxyflavone (4) was achieved. A divergent synthetic strategy was employed, ${ }^{13}$ which allowed access to these biologically interesting compounds in an expedient and step-efficient fashion from readily available starting materials. Notably, multi-milligram quantities of all four natural products were generated, which should provide ample material for screening in biological assays. The divergent strategy is currently being applied to the synthesis of unnatural analogues of compounds $\mathbf{1 - 4}$ to allow the sampling of novel chemical space around these biologically relevant structures. This work, together with the results of biological screening investigations, will be reported in due course.

\section{Acknowledgment}

We thank the Cambridge Commonwealth Trust for the awards of scholarships to T.J.S. and T.H.S. The research leading to these results has received funding from the European Research Council under the European Union's Seventh Framework Programme (FP7/20072013)/ERC grant agreement $n^{\circ}$ [279337/DOS]. The authors also thank AstraZeneca, the European Union (EU), the Engineering and Physical Sciences Research Council (EPSRC), the Biotechnology and Biological
Sciences Research Council (BBSRC), the Medical Research Council (MRC), and the Wellcome Trust for funding. Data accessibility: all data supporting this study are included in the paper and provided as Supporting Information accompanying this paper.

\section{Supporting Information}

Experimental procedures and copies of the ${ }^{1} \mathrm{H}$ NMR and ${ }^{13} \mathrm{C}$ NMR spectra of the synthesized natural products. Supporting information for this

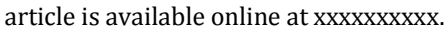

\section{References and Notes}

(1) Detsi, A.; Majdalani, M.; Kontogiorgis, C. A.; Hadjipavlou-Litina, D.; Kefalas, P. Bioorg. Med. Chem. 2009, 17, 8073-8085.

(2) Singh, M.; Kaur, M.; Silakari, O. Eur. J. Med. Chem. 2014, 84, 206 239.

(3) Wang, Z.; Yang, L.; Yang, X.; Zhang, X. Syn. Commun. 2013, 43, 30933114.

(4) Zhang, J.; Fu, X. -L.; Yang, N.; Wang, Q. -A. Sci. World J. 2013, 2013, 1-6.

(5) Kumar, R.; Mohanakrishnan, D.; Sharma, A.; Kaushik, N. K.; Kalia, K.; Sinha, A. K.; Sahal, D. Eur. J. Med. Chem., 2010, 45, 5292-5301.

(6) Ballesteros, J. F.; Sanz, M. J.; Ubeda, A.; Miranda, M. A.; Iborra, S.; Payá, M.; Alcaraz, M. J. Med. Chem. 1995, 38, 2794-2797.

(7) Silva, D. H. S.; Zhang, Y.; Santos, L. A.; Bolzani, V. S.; Nair, M. G. J. Agric. Food Chem. 2007, 55, 2569-2574.

(8) Solankee, A.; Kapadia, K.; Ćirić, M.; Doytchinova, A.; Geronilaki, A. Eur. J. Med. Chem., 2010, 45, 510-518.

(9) Nowakowska, Z. Eur. J. Med. Chem., 2007, 42, 125-137.

(10) Selepe, M. A.; Van Heereden, F. R. Molecules 2013, 18, 4739-4765.

(11) Gao, X.; Yang, L.; Shu, L.; Shen, Y.; Zhang, Y.; Hu, Q. Heterocycles, 2012, 85, 1925-1931.

(12) Shin, H. J.; Nam, J. -W.; Yoon, U. J.; Han, A. -R.; Seo, E. -K. Helvetica Chimica Acta 2012, 95, 240-245.

(13) Sum, T. H.; Sum, T. J.; Stokes, J. E.; Galloway, W. R. J. D.; Spring, D. R. Tetrahedron 2015, 71, 4557-4564.

(14) Shen, Y.; Zhang, J.; Sheng, R.; Dong, X.; He, Q.; Yang, B.; Hu, Y. J. Enzyme Inhib. Med. Chem. 2009, 24, 372-380.

(15) Shen, B.; Löffler, D.; Reischl, G.; Machulla, H. -J.; Zeller, K. -P. J. Fluorine Chem. 2009, 130, 216-224.

(16) Wang, Z. Chapter 13. Algar-Flynn-Oyamada (AFO) Reaction (AlgarFlynn-Oyamada Oxidation). In Comprehensive Organic Name Reactions and Reagents; John Wiley \& Sons, Inc., 2010, 52-56.

(17) Gormley, T. R.; O’Sullivan, W. I. Tetrahedron 1973, 29, 369-373.

(18) Bennett, M.; Burke, A. J.; O’Sullivan, W. I. Tetrahedron 1996, 52, 7163-7178.

(19) Johnson, C. D. Acc. Chem. Res. 1993, 26, 476-482.

(20) Grundon, M. F.; Stewart, D.; Watts, W. E. J. Chem. Soc., Chem. Comm. 1975, 772-773.

(21) Sharma, D.; Makrandi, J. K. J. Heterocycl. Chem. 2014, 51, 18181820.

(22) Agrawal, N. N.; Soni, P. A. Indian J. Chem. 2005, 44B, 2601-2603.

(23) Agrawal, N. N.; Soni, P. A. Indian J. Chem. 2006, 45B, 1301-1303. 